\title{
The Electrical Machining Processes - What Demands will they Face in the Future?
}

\author{
Wilfried KÖNIG*, Fritz KLOCKE*, Rainer LENZEN* \\ (Received on September 11, 1995)
} * Institute of Machine Tools and Production Engineering, RWTH Aachen, Steinbachstraße 53,
D-52074 Aachen, Germany

\begin{abstract}
In many areas, the electrical machining processes face competition from other production processes. If they are to remain viable, they must address themselves to the requirements which will be determined primarily by future products. In addition to the economical aspects which always apply, these requirements also cover new materials, miniaturisation, high environmental compatibility and fast and reliable manufacture.
\end{abstract}

Key words: Electrical machining, state of the art, future outlook, EDM, laser

\section{Introduction}

Developments in the field of production engineering are dominated by far-reaching changes in manufacturing processes. The primary causes behind these changes are requirements for increased accuracy and efficiency of the production processes, the introduction of new materials, the rational production of precision microcomponents in small and medium-sized batches and the increasing consideration of environmental aspects.

It is relatively seldom the case that only one single production process is essentially suitable for a specific machining task. Only after determining and specifying additional parameters, such as machining time, quality of manufacture, reproducibility and economic efficiency, and due consideration of the overall production sequence is the production process finally selected and the production sequence determined.

In this context, the electrical machining processes also become subject to more extensive requirements. In many areas of production, the electrical machining processes furthermore find themselves in competition with other production processes. In view of this situation, specific development of the electrical machining proc- esses in line with future requirements is necessary.

A not unsubstantial number of different machining processes are grouped together today under the term 'electrical machining processes'. Many of these machining processes have grown in importance in recent years, while the importance of some has waned. The most important processes today are electrical discharge machining and laser machining. Others have managed to establish themselves in certain niches. These include ultrasonic machining, for example, which is deployed when fine contours required to be machined from brittle, hard materials.

New processes have recently emerged. These include a large number of rapid prototyping variants, which differ from other processes in that their object is the build-up rather than the removal of material. New workpieces are generated.

As a general rule, the decision as to which production process is to be employed is based on the product to be manufactured, i.e. the product requirements result in the selection of a suitable production process. The electrical machining processes are equally subject to these selection criteria. If they are to remain viable in the future, they must adapt to the prevailing 
product requirements and the extended market and environmental requirements.

\section{Requirements for future products}

The materials employed for products will attain increased importance in the future. This area has been declared a key technology in all industrial nations. Greater emphasis than hitherto will be placed on adapting the material to the product characteristics. But tailor-made materials usually also require special production processes.

For various reasons, work is forging ahead today on the miniaturisation of components. The manufacture of microcomponents and their assembly into microsystems is also regarded as a key technology. With the aid of this technology, material input and energy consumption can be reduced, the environment protected and new, innovative products created. However, there is still a lack of efficient machining processes to manufacture microcomponents and microstructures economically in medium-sized batches.

In our components of the future, the ratio of surface area to volume will continually increase. As a result, the surface will attain increasing importance, as will its characteristics and functions which result from the production process. In the future, therefore, cases and surfaces will be generated and structured specifically with the characteristics which they are to provide in mind.

A third aspect which will have a decisive influence on products is environmental protection. Products will be required to be manufactured and disposed of or recycled in an environmentfriendly manner. Environmentally compatible manufacturing results in completely new requirements with regard to the deployable machining processes.

In addition to the technical requirements relating to future products, the market will also exert an influence on the production processes of the future. For reasons of economic efficiency and in view of the trend towards smaller batch sizes and shorter innovation cycles, production processes will have to become more flexible. Process sequences require to be shortened. These two requirements will increasingly lead to expansions of processes, which may take the form of hybrid processes.

A final aspect, which is also of great importance, is the reliability and reproducibility of a process. The process capability of a production process will become a decisive selection criterion.

The electrical machining processes will, of course, also be judged on the basis of these requirements.

\section{New materials will determine the selection of machining processes}

Important product innovations are emerging from the development of materials. In addition to the familiar materials, new materials designed to incorporate specific properties will become established. New materials, which may also take the form of composites, will impose high requirements on the flexibility of production processes. To enable effective machining of these materials, production information will be required at the planning level. Closer links between the planning and operative levels will be required in future. During their initial phase of application, there is often a lack of knowledge regarding the rough- and finish-machining of many new types of materials. Materials of extremely high hardness, for example, often necessitate new machining processes or machining strategies. In future, we will be required to attach greater emphasis to evolving the advantages of a process and compensating for the disadvantages of a process by incorporating the advantages of other processes. The integration of different production processes to form socalled hybrid processes will become a more common practice in future.

New materials will compel us to seek methods of expanding processes. Electrical discharge 
machining, for example, is a process which can be deployed irrespective of the hardness and stability of a material. A limit is imposed on this process, however, by the need for a minimal level of electrical conductivity. The further development of generators will enable this limit to be shifted in the direction of lesser levels of electrical conductivity. Generators with even higher open-circuit voltages will thus be used in future to machine electrical semi-conductors (e.g. high-performance ceramics).

In addition to electrical discharge machining, other electrical machining processes are also available for the machining of extremely hard materials. Ultrasonic machining, for example, is ideal for machining glass and ceramic materials irrespective of electrical conductivity. As far as the machined materials are concerned, the only limits imposed on this process relate to the brittle-hard properties of the materials. The material removal rates $(30 \mathrm{~mm} / \mathrm{min}$ in glass) are as yet low. The currently replica-based process will become more flexible in the future due to the use of NC technology with simplified sonotrodes.

The laser represents an additional interesting material-removal process for machining extremely hard materials. The development of diode lasers, which will become established alongside gas lasers and solid-state lasers [1] and are considerably smaller and lighter than the conventional beam sources, will provide us with a low-price tool of high flexibility.

The laser can be used to remove and join many types of materials. By means of the hardening, remelting, alloying, dispersion and coating processes, the laser is able to generate localised properties in materials which are adapted to the functions of a component. The production of extremely abrasion-resistant functional zones via the dispersion of carbides such as $\mathrm{TiC}$ into aluminium and steel materials serves as an example here.

In future, the laser will be integrated to an increasing extent in turning and milling ma- chines, in order to utilise the laser's energy to support the cutting process, in addition to the possibilities of modifying the properties of the machined materials.

In connection with the machining of hard materials, mention must also be made of the classical machining processes of turning and milling. Thanks to both new tool materials and improved machine components, it is now possible to machine extremely hard steels ready for installation. This trend will continue. Turning and milling today represent strong and growing rival processes in many fields of tool- and mould-making. The electrical machining processes will have to compete with these processes.

\section{Miniaturisation of products}

Microsystems engineering, which involves the integration of various mechanical, optical and electrical functions, is regarded as a key technology of tomorrow. With the aid of this technology, material input and energy consumption can be reduced, the environment protected and new, innovative products created. Examples here are applications in the field of medical technology (dosing devices for the supply of medicines in the body) or microsensors in traffic engineering. To date, however, there is still a lack of efficient machining processes to produce microcomponents and microstructures. The production of such components and structures using the established mass-production processes for semiconductor components is technically and economically unfeasible. On account of their specific mechanisms of action, electrical machining processes would appear highly suitable for the machining of microcomponents, however. High-precision wire cutting provides an example here.

Here again, the laser technologies possess areas of potential which have yet to be exhausted. Certain laser modifications (excimer laser and $\mathrm{Nd}$ :YAG laser) enable the generation of extremely precise structures in the micrometre range. Laser permits both fast machining and 
the machining of new materials. Further studies are required in this area, however.

Electrical discharge machining (EDM) is a suitable machining process for the production of microcomponents and microstructures. In view of its two outstanding characteristics - virtually force-free machining and independence of the hardness and stability of the workpiece material - electrical discharge machining would appear to be predestined for such applications. In order to utilise EDM for this task, the entire system requires to be reconfigured, however. It will not be sufficient to "miniaturise" the existing and deployed systems.

As a result of the general trend towards the miniaturisation of components, the ratio of surface area to volume continues to rise, with a subsequent increase in the importance of the surface's form and characteristics. Surface zone properties induced by the machining process will require increased attention. For electrical discharge machining, this will mean a combination of the EDM and ECM processes. On one and the same machine this hybrid process will erode the component and then remove the affected surface zone in a subsequent brief operation.

\section{Products will have to be environmentally compatible}

Future products will have to be produced and disposed of in an environment-friendly manner and will further be required to behave in an environmentally compatible manner during use. Analysis of the environmental effects during manufacture enables the environmentally hazardous aspects of production processes to be eliminated or reduced. To this end, the attendant hazards must first of all be established and evaluated. An analysis of the causes then requires to be carried out. Once the causes are known, appropriate concepts to solve the problems concerned can be evolved. The object of these concepts is to reduce or avoid the hazards via the isolation, modification or substitution of processes [2]. In the case of electrical machin- ing processes, further development work is required with regard to the aspect of environmentally compatible manufacture.

For the laser, this means that the emissions which occur in the process require to be identified, reduced and isolated. But other electrical machining processes will also have to comply more effectively with the growing environmental requirements, if they are to avoid losing importance in the future as a result of statutory provisions. A case in point here for electrical discharge machining is the working media employed during machining. Water-based working media will become increasingly more widespread. With regard to ECM processes, the employed electrolyte represents a critical component in terms of environmental compatibility. Further development of the treatment plants to separate heavy metals from the pure electrolyte is necessary here.

The strong influence of statutory provisions on production operations will continue to grow. In the European Union statutory provisions have now been created which, among other things, compel machine tool manufacturers to observe certain limits with regard to electromagnetic emission. It may be assumed that the number of laws and provisions will continue to grow in the future. This situation will also require due consideration in the further development of electrical machining processes.

\section{Swift launching of high-quality, reproducible products}

The further globalisation of markets and increasingly keener competition will compel companies to design and manufacture new products more quickly.

In order to shorten the development phase, the design, geometry and required functions of a product must be known as early as possible during the development stage. The rapid prototyping processes have emerged as a result of this requirement. A large number of different processes have developed in recent years [3]. 
The primary materials employed today are waxes, plastics, paper and several ceramic materials. The prime aim of all rapid prototyping processes is to produce a physical model from a CAD model in the shortest of time. Despite the large number of modifications to these processes, a common feature of them all is that, as opposed to material being removed, (pilot) products "grow" via the addition of material. Apart from enabling visual assessment of the design, functional and technical prototypes span a far broader range of applications, whereby functional prototypes are employed to assess the technical functions of a design. The material properties of functional prototypes should be as close as possible to those of the component planned for series production, which means that metallic rapid prototyping parts will be required in the future [4]. The maximum attainable accuracies currently lie within the range of $0.1 \%$ of the dimension to be manufactured, whereby the surface quality is directly dependent on the attainable layer thickness. Improved dimensional accuracy and surface quality are desirable, whereby considerably greater attention must be paid to the possibility of simultaneously altering the metallurgical structure of the metal in accordance with the specific requirements of the product.

The improvement of production quality and reliability requires increasingly more extensive measures to optimise the production processes. Whereas the machine operator's manual skill was originally adequate to ensure sufficient reliability for high product quality, the increasing degree of automation in conjunction with shorter machining times now requires increased safety and reliability in production processes and, subsequently, the use of sensors to monitor processes. Furthermore, new strategies are needed to machine complex components. Such strategies require the controlled correction of process variables and will also render it essential for technological knowledge to be incorporated into process design on a much broader scale than currently applies.
As a result of the continually increasing capacity of computer systems, the volume of process variables which are scanned for control purposes and fed back to the machine is subject to constant growth. However, not all the variables influencing a process can be determined with sufficient accuracy, resulting in a certain process uncertainty or unsharpness. Efforts are made to further minimise this residual uncertainty with the aid of new control algorithms, such as fuzzy logic. The next step will involve coupling neural networks with fuzzy logic, so as to further enhance process reliability.

\section{Summary}

In many areas, the electrical machining processes face strong competition from other production processes. In order to remain viable in the future, they must undergo further development in accordance with the requirements which will apply to future products. The new materials, geometric dimensions, high accuracy, environmental conditions and the safe and fast manufacture of products all play a decisive role in this context. A certain scope of preliminary work has already been carried out, but further efforts will be necessary, whereby close cooperation between the research and operational levels is required, in order to transform theoretical knowledge swiftly into marketable products. Provided that this work is carried out in an effective manner, the electrical machining processes are set to come into their own in the future as more efficient processes within a reduced production sequence.

\section{Bibliography}

[1] N.N.: Hochleistungsdiodenlaser - eine neue Dimension in der Fertigungstechnik, Blech, 3/95

[2] Weck, M.; Eversheim, W.; König, W.; Pfeifer, T.: Fertigung im umwelttechnischer Verantwortung, Wettbewerbsfaktor Produktionstechnik, Düsseldorf. Published by VDI-Verlag, 1990 
[3] Pfeifer, T.; Weck, M.; Eversheim, W.; König, W.: Strategien und Verfahren zur Fertigung von Prototypen, Wettbewerbsfaktor Produktionstechnik - Aachener Perspektiven, Düsseldorf. Published by VDI-Verlag, 1993
[4] Hartung, St.: Rapid Prototyping auf dem Weg zum Metall, Produktion, issue 25/26, 1995 